\title{
Studies on placentae and infants from women vaccinated for smallpox during pregnancy
}

\author{
P. WENTWORTH
}

From the Department of Pathology, Welsh National School of Medicine, Cardiff

SYNOPSIS Sixty-five placentae from women who had been vaccinated while pregnant were examin\& for the presence of the inclusion bodies of vaccinia. Also suspensions from 40 of the placentae wẹpe inoculated into the chorio-allantoic membrane of chick embryos in an attempt to culture any vaccinta virus present. Both these studies gave negative results.

The results of the pregnancies of these 65 women showed no increase in the incidence of abortion, stillbirth, or congenital abnormalities, over the unvaccinated women in the overall series.

Ever since the observation by Gregg (1941) of a high incidence of congenital defects of the eye in children born to women who had contracted rubella in pregnancy, interest has been stimulated in infection as a probable aetiological factor in congenital abnormality, abortion, and stillbirth. Recent views on this subject have been summarized by Medearis (1964). Interest has similarly been aroused in the artificial introduction into the body of virus products by immunization procedures during pregnancy (Greenberg, Yankauer, Krugman, Osborn, Ward, and Dancis, 1949; Zellweger, 1957; Bourke and Whitty, 1964).

A smallpox outbreak occurred in Cardiff between 13 January 1962 and mid-April 1962, and 139,000 people were vaccinated as a result. At the time a continuous series of placentae was being collected from patients delivered at the Maternity Hospital and the opportunity was taken to examine the placentae in this series from those women who had been vaccinated while pregnant.

\section{MATERIAL AND METHODS}

The placentae collected were from 65 women who had been vaccinated during the first trimester of pregnancy. The placentae were all collected within 24 hours of delivery, the actual times varying from within one half to 23 hours after delivery. Placentae from two other women who contracted rubella, one in the first trimester and the other in the second trimester, were also examined.

Any gross placental abnormality, such as circumvallate type or succenturiate lobes, and vascular anomalies, such as single umbilical artery or velamentous insertion of the cord, were noted.

Histological sections were taken from the chorionic plate, the basal plate, and the intermediate zone of $\overleftarrow{w_{1} 5}$ placentae, and stained by the phloxin-tartrazine method for inclusion bodies (Lendrum, 1947). This was done in attempt to demonstrate the presence of any intracy 10 plasmic inclusion bodies, which are found in tissues infected with vaccinia.

In 40 of the 65 placentae portions were taken from the chorionic plate, basal plate, and intermediate zone ahd immediately placed in a deep freeze at $-25^{\circ} \mathrm{C}$. St? pensions of this material were subsequently inoculated on to the chorio-allantoic membrane of 10-12-day-odd chick embryos in an attempt to culture any vaccinia viris present.

The duration of pregnancy, outcome of the pregnanoy (whether abortion, stillbirth, congenital abnormality, normal live birth) were noted in each case, the approfmate stage of the pregnancy in weeks when vaccination was performed, and any maternal reactions to the vaccination.

Four mothers had been vaccinated repeatedly because earlier attempts did not take. All the other mothers kngw the date within one week when they had been vaccinated. All said that the vaccination had been successful 공s judged by the doctor who had performed the vaccination when they returned to him 48 hours later for it to ige checked. All these were re-vaccinations, primary vaccinations having been done anything from one to 20 ye previously. It was not possible to check these women vaccination scars, but from the history vaccination was assumed to be successful.

\section{RESULTS}

The stage of the pregnancy when vaccination ws performed and the duration of pregnancy are listod in Tables I and II.

Gross examination of the placentae showed the 18 were circummarginate in type, one was circu䭪vallate, two had succenturiate lobes, two had 
TABLE I

STAGE OF THE PREGNANCY WHEN VACCINATION WAS PERFORMED

Approximate Stage of Pregnancy (weeks) when Vaccinated

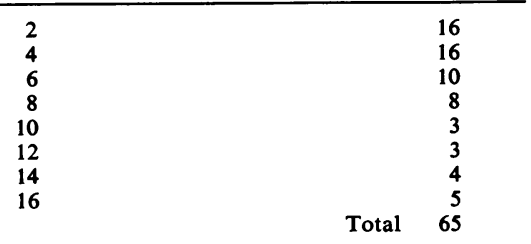

TABLE II

DURATION OF PREGNANCY

Duration of Pregnancy (weeks)

No. of Cases

28
37
38
39
40
41
42
43

${ }^{1}$ Denotes inclusion of a stillbirth.

$\begin{array}{cc} & 1^{1} \\ & 6 \\ & 4 \\ & 6 \\ & 31^{1} \\ & 9 \\ & 6 \\ & 2 \\ \text { Total } & 65\end{array}$

aneurysms of the chorionic vessels, and one a battledore insertion of the cord. Four placentae required manual removal. All these features occurred only with the expected frequency for any like series of placentae (Wentworth, 1965).

Histological examination of the placentae for inclusion bodies and chorio-allantoic membrane inoculation for virus culture both gave negative results.

There were no abortions in the series. There were two stillbirths: one, at approximately 28 weeks, was a male hydropic foetus weighing $3 \mathrm{lb} .11 \mathrm{oz}$., and the mother was subsequently discovered to have a high titre of anti-D in her serum. The other stillbirth occurred at 40 weeks and was a male foetus weighing $6 \mathrm{lb} .11 \mathrm{oz}$. The foetal heart was last heard a few minutes before delivery. The mother was vaccinated at approximately the second week of pregnancy.

There was only one congenital abnormality in the series, a female infant weighing $7 \mathrm{lb} .2 \mathrm{oz}$. with spina bifida. The mother had been vaccinated at approximately the fourth week of pregnancy. All other pregnancies resulted in normal live births. One woman had a severe reaction following vaccination during the tenth week of pregnancy. She had severe headache, rigors, and delirium, and a rash on her lips, arms, and legs. She delivered a normal healthy male infant weighing $7 \mathrm{lb} .11 \mathrm{oz}$. at the 38th week.

The mothers who had rubella at the 11 th and 23 rd weeks of pregnancy respectively both delivered normal, healthy, full-term infants, and the placentae were normal.

\section{DISCUSSION}

Dixon (1962) states that 'although there is a viraemia during vaccination the foetus does not appear to be affected as in rubella'. He went on to say, however, that because of the increased risk of stillbirth, vaccination was best avoided during the first three months of pregnancy.

Bourke and Whitty (1964) confirmed Dixon's view that the vaccinia virus was without teratogenic effect, when they found that the incidence of congenital malformations among children whose mothers were vaccinated during pregnancy was not appreciably greater than average. They also found that the incidence of abortions and stillbirths was also no greater than the average. Greenberg et al. (1949) produced similar findings from their study following the mass vaccination programme in New York City early in 1947. These findings are supported by the present study in which the incidence of congenital abnormality, abortion, and stillbirth was also no greater than the local average.

However, occasional fatal cases of generalized vaccinia of the foetus are reported (MacDonald and MacArthur, 1953; Tucker and Sibson, 1962; Entwistle, Bray, and Laurence, 1962). The placenta from the case of Entwistle et al. showed numerous yellowish areas of necrosis and in the cells surrounding these areas there were typical large eosinophilic cytoplasmic inclusions. Similar findings in the placenta have been described by Garcia (1963). As no foetus was affected in the present series the failure to find these inclusions or to grow the virus was not surprising.

This fatal vaccinia of the foetus can occur when the mother is vaccinated during the middle of pregnancy and not only during the first three months (Entwistle et al., 1962; Wielenga, Van Tongeren, Ferguson, and Van Rijssel, 1961). Because of the occasional occurrences of these cases, the negative findings of the present series do not alter the dictum that 'unless the indications for vaccinating the mother are very strong indeed it is wiser to avoid vaccination against smallpox at any time during pregnancy' (Brit. med. J., 1964, leading article).

It is hoped that this study is a useful response to the observation of Hardy (1965) that remarkably little information is available about the effectiveness of the placenta as a protective barrier and its role in transplacental infections.

I would like to thank Professor A. S. Duncan for permission to study the patients under his care; Professor J. 
Gough for his encouragement and support throughout; and Dr. A. D. Evans for undertaking the viral studies.

\section{REFERENCES}

Bourke, G. J., and Whitty, R. J. (1964). Brit. med. J., 1, 1544. British Medical Journal (1964). Leading article, 1, 1524.

Dixon, C. W. (1962). Ibid., 1, 1262.

Entwistle, D. M., Bray, P. T., and Laurence, K. M. (1962). Ibid., 2, 238.

Garcia, A. G. P. (1963). Pediatrics, 32, 895.
Greenberg, M., Yankauer, A. Jr., Krugman, S., Osborn, J. J., W R. S., and Dancis, J. (1949). Ibid., 3, 456.

Gregg, N. McA. (1941). Trans. Ophthal. Soc. Aust., 3, 35.

Hardy, J. B. (1965). Amer. J. Obstet. Gynec., 93, 1052.

Lendrum, A. C. (1947). J. Path. Bact., 59, 399.

M. (1953). Arch. Dis. Child 311 .

Medearis, D. N. Jr. (1964). Amer. J. Obstet. Gynec., 90, 1140.

Tucker, S. M., and Sibson, D. E. (1962). Brit. med. J., 2, 237.

Wentworth, P. (1965). M.D. Thesis, Welsh National Schoo廑of Medicine, Cardiff, Wales.

Wielenga, G., Van Tongeren, H. A. E., Ferguson, A. H., and Dan Rijssel, T.G. (1961). Lancet, 1, 258.

Zellweger, H. (1957). Helv. paediat. Acta, 12, 563. 